J. Clin. Chem. Clin. Biochem.

Vol. 17, 1979, pp. 517-522

\title{
Falsch positive Werte bei der Vielfachanalyse: Eine Erhebung an Referenz- und Patientenkollektiven
}

\author{
Von K. Harm, W. Rehpenning, Astrid Domesle und K. D. Voigt \\ Zentrallabor der Medizinischen Kliniken (Prof. Dr. K. D. Voigt) und Abteilung für Mathematik in der Medizin \\ (Prof. Dr. J. Berger) des Universitäts-Krankenhauses Hamburg-Eppendorf
}

(Eingegangen am 18. September 1978/4. April 1979)

Zusammenfassung: An jeweils zwei Patienten- und Referenzkollektiven wurde die bei der 12-fach-Analyse auftretende Anzahl von falsch positiven Werten ermittelt. Dabei zeigte sich, daß der wirklich vorhandene Anteil an falsch positiven Werten statistisch signifikant unter dem nach der Binomialverteilung errechneten liegt. Als Ursache für diese Diskrepanz zwischen Theorie und Realität sind sowohl zum Teil ausgeprägte Korrelationen der zu einem Profil zusammengestellten Parameter untereinander als auch deutliche Abweichungen von der Normalverteilung anzusehen. Die Ergebnisse zeigen, daß die Anwendung der Binomialverteilung zu Aussagen führt, die signifikant von den tatsächlichen Verhältnissen abweichen.

\section{Falsely positive values in multi-channel analysis: An inquiry into reference and patient groups}

Summary: The number of falsely positive values occurring in 12-channel analysis was determined in two groups of patients and reference individuals. It revealed that the portion of falsely positive values actually found was statistically significant beyond that calculated on the assumption of a binomial distribution. Partly distinct correlations of the parameters combined to a profile as well as clear deviations from the normal distribution have to be taken into consideration as reasons for this discrepancy between theory and reality. The results show that the application of the binomial distribution leads to statements which significantly differ from the conditions actually present.

\section{Einfïhrung}

In den letzten Jahren wurde das Problem des Auftretens von falsch positiven Werten, d. h. Werten, die durch die Definition der Referenzbereiche als Bereiche, in denen 95\% der bei Gesunden gemessenen Werte liegen, von verschiedenen Gesichtspunkten her behạndelt $(1,2,3)$. Dabei wurde manchmal mehr emotional als sachlich argumentiert, insbesondere wurden falsch positive Werte speziell der Mehrkanalanalyse mit Autoanalyzern angelastet, obwohl dieses Phänomen ein ganz allgemeines ist, das nư bei Flow-Systemen besonders evident wird.

Büttner (4) hat die Diskussion wieder auf den Boden der Tatsachen zurückgeführt und mathematische Kriterien zur Beurteilung des diagnostischen Wertes klinisch-chemischer Untersuchungen angegeben, wobei auch auf die Verhältnisse bei der Mehrfachanalyse eingegangen wurde.

În einer kürz̄lich durchgeführten orientierenden Studie konnten wir zeigen, daß die tatsächlich beobachteten Häufigkeiten falsch poșitiver Werte nicht unbedingt mit den theoretisch zu erwartenden übereinstimmen $(5,6)$. Wir hielten es daher für notwendig, in einer Erhebung an mehreren, zum Teil grộn Kollektiven die Häufigkeị des
Auftretens falsch positiver Werte experimentell zu überprüfen. Natürlich hängt die Entscheidung darüber, ob ein Wertesatz falsch positiv ist, von der Definition und auch vom zu Grunde gelegten Kollektiv ab. Wir legen hier die univariate Betrachtungsweise zu Grunde, d. h. wir bezeichnen einen Wertesatz als falsch positiv bezogen auf das untersuchte Kollektiv, wenn mindestens ein Parameter aus dem entsprechenden univariaten Referenzbereich herauusfällt. Bei statistisch unabhängigen Parametern wäre dann die Wahrscheinlichkeit für das Auftreten falsch positiver Wertesätze nach der Binomialformel zu berechnen (4).

Im folgenden soll an der Realität geprüft werden, welchen Einfluß das Vorliegen von Korrelationen zwischen den Parametern auf die Häufigkeit des Auftretens falsch positiver Werte hat.

\section{Material und Methoden}

Es wurden vier Kollektive ausgewertet, zwei Referenzund zwei Patientenkollektive. Bei den Referenzkollek- 
tiven handelte es sich um 313 bzw. 342 Ärzte, Technische Assistentinnen und Pflegepersonen des UniversitätsKrankenhauses Eppendorf, bei denen anläßlich der in den Jahren 1976 und 1977 durchgeführten routinemäßigen Personaluntersuchungen klinisch-chemische Profile angefordert wurden. $\mathrm{Ob}$ alle Personen dieser Kollektive wirklich gesund waren, läßt sich natürlich nicht mit letzter Sicherheit sagen, für die hier untersuchte Fragestellung ist dieses auch nicht unbedingt erforderlich. Das Alter dieser Referenzpersonen lag zwischen etwa 20 und 65 Jahren. Durch Namenidentifikation wurde sichergestellt, daß jede Person nur einmal in die Rechnung einging.

Es standen uns etwa 60000 unausgewählte Patienten der Jahre 1976 und 1977 für die Auswertung zur Verfügung. $D a$ wir uns naturgemäß auf Teilkollektive beschränken mußten, sind wir folgendermaßen vorgegangen: Die Patientendaten wurden in zeitlicher Reihenfolge in Gruppen von etwa 1500 bis 5000 Personen auf Magnetbändern gespeichert. Für die Rechnungen haben wir willkürlich zwei Untergruppen herausgegriffen. Durch Abfrage über die Aufnahmenummer konnten Mehrfachmessungen eliminiert werden. Dieses war aus technischen Gründen aber nur innerhalb der jeweiligen Untergruppe möglich. Allerdings ist nicht auszuschließen, daß dennoch ein Patient mehrfach im Kollektiv enthalten ist, da bei erneuten Krankenhausaufnahmen innerhalb des gleichen Kalenderjahres jeweils eine neue Aufnahmenummer vergeben wird. Durch dieses Verfahren ergaben sich 4763 bzw. 3423 Datensätze.

Sämtliche Profile wurden mit einem Technicon Autoanalyzer SMA 12/60 erstellt. Bei folgenden Kanälen wurden die im Handbuch des Herstellers (7) angegebenen Bestimmungsmethoden benutzt: Natrium, Kalium (Flammenphotometrie), Chlorid (Quecksilberthiocyanat-Methode), Gesamt-Eiweiß (Biuret-Reaktion), anorganischer Phosphor (Molybdänblau-Reaktion), Harnstoff-Stickstoff (Diacetylmonoxim-Reaktion), Calcium (Cresolphthalein-Methode), Kreatinin (Jaffé-Reaktion), Bilirubin (Azobilirubin-Reaktion nach Jendrassik \& Gróf), Harnsäure (Phosphorwolframsäure-Methode). Die Bestimmung des Albumins erfolgte mit der Bromkresolgrün-Methode (8), die des Cholesterins 1976 mit der Liebermann-Burchard-Reaktion (7), 1977 mit der Cholesterinoxidase-Phenol-Aminophenazon-Methode (9). Die Meßwerte wurden on-line vom Telefünken-Rechner TR 86 des Systems ELIAS (10) erfaßt. Nach der Wandlung in eine für den Großrechner TR 440 des Rechenzentrums der Universität Hamburg lesbare Form erfolgte auf diesem die Auswertung der Daten mit Hilfe von FORTRAN-Programmen.

Zur Kennzeichnung der Verteilungen sowohl der Referenzpersonen als auch der Patienten hielten wir es für bestgeeignet, Quantile (11) zu berechnen, weil die Verteilungen zum Teil sehr schief sind, großen positiven Exzeß aufweisen (12) und weil insbesondere bei den Patientendaten extreme Werte vorkommen, die man nicht ohne weiteres als Ausreißer betrachten darf. Diese Werte sind im allgemeinen als real anzusehen und somit Bestandteil des Kollektivs. Weiterhin wurden für alle Kollektive die Matrizen der Rang-Korrelationen berechnet (13). $\mathrm{Da}$ die Speicherkapazität des Großrechners beschränkt ist, mußten wir uns hierbei auf maximal 750 Fälle beschränken.

Das Auftreten falsch positiver Werte haben wir dahingehend differenziert, daß wir abzählten, wie viele Parameter eines Profils außerhalb des durch die Quantile definierten 95\%-Bereiches lagen und daß wir diese Anteile mit den nach der Binomialverteilung zu erwartenden verglichen. Hierbei ist zu beachten, daß die "falsch positiven Werte" jeweils am zugehörigen Kollektiv definiert sind. Außerdem wurde noch zum Vergleich zwischen einem Referenz- und Patientenkollektiv berechnet, wie viele Patientenwerte (parameterspezifisch) unterhalb bzw. oberhalb der Quantile des Referenzkollektivs liegen.

\section{Ergebnisse und Diskussion}

Die Quantile der Verteilungen in den beiden Referenzkollektiven sind in den Tabellen 1 und 2 dargestellt. Der Vergleich dieser statistischen Kenngrößen zeigt, daß nur geringfügige Unterschiede zwischen den beiden Jahrgängen bestehen. Sie sind am ausgeprägtesten in den Medianen bei Natrium, Chlorid, Kreatinin und Bilirubin. Weiterhin zeigt die Betrachtung der angegebenen Quantile, daß die Werte fast aller Kenngrößen nicht normalverteilt sind, so daß der 95\%-Referenzbereich sich nicht als $\overline{\mathrm{x}} \pm 2$ s, sondern nur als $Q_{0,025}$ bis $Q_{0,975}$ korrekt angeben läßt.

Die entsprechenden Quantile für die Verteilungen der beiden Patientenkollektive sind in den Tabellen 3 und 4 dargestellt. Auch hier findet man eine erstaunlich gute Übereinstimmung der Quantile beider Jahrgänge, was für eine sehr gute Stabilität sämtlicher Verteilungen spricht. Der Vergleich der Patienten-mit den Referenzkollektiven zeigt, daß die Verteilungen der Patientenwerte eine deutlich ausgeprägtere Asymmetrie aufweisen, ein Phänomen, dem u. a. eine gewisse Bedeutung bei dem Versuch einer Identifizierung falsch positiver Werte zukommt.

In Tabelle 5 ist in Prozent angegeben, wie häufig Profile außerhalb der $95 \%$-Referenzbereiche in den beiden Referenzkollektiven liegen. Die aufgeführten Zahlen zeigen einmal, daß nur geringfugige Unterschiede zwischen den beiden Kollektiven bestehen, sie demonstrieren aber auch ganz eindeutig, daß der wirklich vorhandene Gesamtanteil an falsch positiven Werten deutlich unter dem nach der Binomialverteilung errechneten liegt. Dabei ergeben sich die Ëinzelanteile der zu erwartenden falsch positiven Resultate bei der 12-fach-Analyse 
Tab. 1. Quantile der Verteilungen im Referenzkollektiv $1976(\mathrm{~N}=313)$

\begin{tabular}{|c|c|c|c|c|c|c|c|c|}
\hline Komponente & Einheit & $Q_{0,005}$ & $Q_{0,010}$ & $Q_{0,025}$ & $\begin{array}{c}\text { Median } \\
\mathrm{Q}_{0,500}\end{array}$ & $Q_{0,975}$ & $Q_{0,990}$ & $Q_{0,995}$ \\
\hline $\begin{array}{l}\text { Natrium } \\
\text { Kalium } \\
\text { Chlorid } \\
\text { Gesamt-Eiweiß } \\
\text { Albumin } \\
\text { Anorg. Phosphor } \\
\text { Cholesterin } \\
\text { Harnstoff-N } \\
\text { Calcium } \\
\text { Kreatinin } \\
\text { Bilirubin } \\
\text { Harnsäure }\end{array}$ & $\begin{array}{r}\mathrm{mmol} / 1 \\
\mathrm{mmol} / 1 \\
\mathrm{mmol} / 1 \\
\mathrm{~g} / 1 \\
\mathrm{~g} / 1 \\
\mathrm{mmol} / 1 \\
\mathrm{mmol} / 1 \\
\mathrm{mmol} / 1 \\
\mathrm{mmol} / 1 \\
\mu \mathrm{mol} / 1 \\
\mu \mathrm{mol} / 1 \\
\mu \mathrm{mol} / 1\end{array}$ & $\begin{array}{c}136,3 \\
3,519 \\
97,73 \\
59,06 \\
36,69 \\
0,589 \\
3,733 \\
2,293 \\
2,199 \\
54,37 \\
4,136 \\
171,5\end{array}$ & $\begin{array}{c}137,4 \\
3,552 \\
97,83 \\
61,69 \\
37,59 \\
0,618 \\
3,779 \\
2,573 \\
2,225 \\
57,21 \\
4,136 \\
177,6\end{array}$ & $\begin{array}{c}138,1 \\
3,607 \\
98,47 \\
64,40 \\
38,97 \\
0,708 \\
3,951 \\
2,975 \\
2,255 \\
60,15 \\
4,385 \\
191,0\end{array}$ & $\begin{array}{c}142,3 \\
4,273 \\
103,7 \\
72,11 \\
44,18 \\
1,085 \\
5,334 \\
5,214 \\
2,439 \\
88,02 \\
10,53 \\
307,3\end{array}$ & $\begin{array}{c}145,9 \\
5,036 \\
109,2 \\
79,75 \\
49,40 \\
1,419 \\
7,334 \\
8,334 \\
2,593 \\
118,3 \\
27,77 \\
469,6\end{array}$ & $\begin{array}{c}147,7 \\
5,119 \\
110,6 \\
81,68 \\
50,08 \\
1,472 \\
8,149 \\
9,008 \\
2,643 \\
127,2 \\
35,89 \\
526,1\end{array}$ & $\begin{array}{c}148,1 \\
5,226 \\
110,9 \\
83,25 \\
51,09 \\
1,558 \\
8,306 \\
9,784 \\
2,688 \\
146,5 \\
44,46 \\
540,0\end{array}$ \\
\hline
\end{tabular}

Tab. 2. Quantile der Verteilungen im Referenzkollektiv $1977(\mathrm{~N}=342)$

\begin{tabular}{|c|c|c|c|c|c|c|c|c|}
\hline Komponente & Einheit & $Q_{0,005}$ & $Q_{0,010}$ & $Q_{0,025}$ & $\begin{array}{l}\text { Median } \\
Q_{0,500}\end{array}$ & $Q_{0,975}$ & $Q_{0,990}$ & $Q_{0,995}$ \\
\hline $\begin{array}{l}\text { Natrium } \\
\text { Kalium } \\
\text { Chlorid } \\
\text { Gesamt-Eiweiß } \\
\text { Albumin } \\
\text { Anorg. Phosphor } \\
\text { Cholesterin } \\
\text { Harnstoff-N } \\
\text { Calcium } \\
\text { Kreatinin } \\
\text { Bilirubin } \\
\text { Harnsäure }\end{array}$ & $\begin{array}{r}\mathrm{mmol} / 1 \\
\mathrm{mmol} / 1 \\
\mathrm{mmol} / 1 \\
\mathrm{~g} / 1 \\
\mathrm{~g} / 1 \\
\mathrm{mmol} / 1 \\
\mathrm{mmol} / 1 \\
\mathrm{mmol} / 1 \\
\mathrm{mmol} / 1 \\
\mu \mathrm{mol} / 1 \\
\mu \mathrm{mol} / 1 \\
\mu \mathrm{mol} / 1\end{array}$ & $\begin{array}{c}136,9 \\
3,191 \\
95,82 \\
56,87 \\
33,47 \\
0,756 \\
2,570 \\
2,540 \\
2,120 \\
57,83 \\
2,483 \\
159,0\end{array}$ & $\begin{array}{c}138,3 \\
3,507 \\
96,96 \\
63,34 \\
36,51 \\
0,769 \\
3,403 \\
3,029 \\
2,221 \\
62,73 \\
2,820 \\
171,0\end{array}$ & $\begin{array}{c}139,8 \\
3,674 \\
99,06 \\
64,72 \\
38,68 \\
0,802 \\
3,693 \\
3,239 \\
2,263 \\
68,95 \\
3,760 \\
181,0\end{array}$ & $\begin{array}{c}143,6 \\
4,262 \\
104,8 \\
71,95 \\
43,87 \\
1,132 \\
5,441 \\
5,313 \\
2,452 \\
92,42 \\
9,775 \\
302,4\end{array}$ & $\begin{array}{c}150,4 \\
5,126 \\
111,6 \\
79,96 \\
48,44 \\
1,504 \\
8,443 \\
9,187 \\
2,632 \\
130,4 \\
23,49 \\
485,5\end{array}$ & $\begin{array}{c}151,6 \\
5,299 \\
112,3 \\
81,53 \\
49,17 \\
1,583 \\
9,800 \\
9,875 \\
2,684 \\
139,9 \\
32,15 \\
507,0\end{array}$ & $\begin{array}{c}151,8 \\
5,361 \\
114,3 \\
81,93 \\
49,85 \\
1,611 \\
12,04 \\
9,893 \\
2,724 \\
182,1 \\
40,91 \\
554,8\end{array}$ \\
\hline
\end{tabular}

Tab. 3. Quantile der Verteilungen im Patientenkollektiv $1976(\mathrm{~N}=4763)$

\begin{tabular}{|c|c|c|c|c|c|c|c|c|}
\hline Komponente & Einheit & $Q_{0,005}$ & $Q_{0,010}$ & $Q_{0,025}$ & $\begin{array}{l}\text { Median } \\
Q_{0,500}\end{array}$ & $Q_{0,975}$ & $Q_{0,990}$ & $Q_{0,995}$ \\
\hline $\begin{array}{l}\text { Natrium } \\
\text { Kalium } \\
\text { Chlorid } \\
\text { Gesamt-Eiweiß. } \\
\text { Albumin } \\
\text { Anorg. Phosphor } \\
\text { Cholesterin } \\
\text { Harnstoff-N } \\
\text { Calcium } \\
\text { Kreatinin } \\
\text { Bilirubin } \\
\text { Harnsäure }\end{array}$ & $\begin{array}{r}\mathrm{mmol} / 1 \\
\mathrm{mmol} / 1 \\
\mathrm{mmol} / \mathrm{l} \\
\mathrm{g} / \mathrm{l} \\
\mathrm{g} / \mathrm{l} \\
\mathrm{mmol} / \mathrm{l} \\
\mathrm{mmol} / 1 \\
\mathrm{mmol} / 1 \\
\mathrm{mmol} / 1 \\
\mu \mathrm{mol} / 1 \\
\mu \mathrm{mol} / \mathrm{l} \\
\mu \mathrm{mol} / 1\end{array}$ & $\begin{array}{c}125,7 \\
3,108 \\
84,01 \\
43,17 \\
22,28 \\
0,430 \\
2,474 \\
1,975 \\
1,701 \\
36,67 \\
3,196 \\
113,4\end{array}$ & $\begin{array}{c}129,4 \\
3,252 \\
87,36 \\
48,77 \\
24,71 \\
0,507 \\
2,803 \\
2,251 \\
1,874 \\
39,61 \\
3,384 \\
132,3\end{array}$ & $\begin{array}{c}132,8 \\
3,463 \\
91,64 \\
54,04 \\
27,82 \\
0,649 \\
3,260 \\
2,686 \\
1,997 \\
48,41 \\
3,948 \\
155,9\end{array}$ & $\begin{array}{c}142,2 \\
4,362 \\
103,1 \\
71,34 \\
41,51 \\
1,130 \\
5,391 \\
5,648 \\
2,419 \\
86,55 \\
9,587 \\
311,2\end{array}$ & $\begin{array}{c}148,1 \\
5,716 \\
110,6 \\
82,50 \\
48,89 \\
1,746 \\
8,400 \\
21,92 \\
2,706 \\
225,7 \\
38,05 \\
557,1\end{array}$ & $\begin{array}{c}149,7 \\
6,658 \\
113,5 \\
84,83 \\
50,13 \\
2,012 \\
9,250 \\
30,28 \\
2,821 \\
620,4 \\
59,82 \\
648,6\end{array}$ & $\begin{array}{c}151,2 \\
7,449 \\
115,0 \\
87,56 \\
51,03 \\
2,168 \\
9,960 \\
34,75 \\
2,974 \\
794,0 \\
79,63 \\
678,4\end{array}$ \\
\hline
\end{tabular}

Taḅ. 4. Quantile der Verteilungen ịm Patientenkollektiv $1977(\mathrm{~N}=3423)$

\begin{tabular}{|c|c|c|c|c|c|c|c|c|}
\hline Komponente & Einheit & $Q_{0,005}$ & $Q_{0,010}$ & $Q_{0,025}$ & $\begin{array}{l}\text { Median } \\
Q_{0,500}\end{array}$ & $Q_{0,975}$ & $Q_{0,990}$ & $Q_{0,995}$ \\
\hline $\begin{array}{l}\text { Natrium: } \\
\text { Kalium } \\
\text { Chlorid } \\
\text { Gesamt-Eiweiß } \\
\text { Albumin } \\
\text { Anorg. Phosphor } \\
\text { Cholestërin } \\
\text { Harnstoff-N } \\
\text { Calcium } \\
\text { Kreatinin } \\
\text { Bilirubin } \\
\text { Harnsäure }\end{array}$ & $\begin{array}{r}\mathrm{mmol} / 1 \\
\mathrm{mmol} / 1 \\
\mathrm{mmol} / \mathrm{l} \\
\mathrm{g} / \mathrm{l} \\
\mathrm{g} / \mathrm{l} \\
\mathrm{mmol} / \mathrm{l} \\
\mathrm{mmol} / \mathrm{i} \\
\mathrm{mmol} / 1 \\
\mathrm{mmol} / \mathrm{l} \\
\mu \mathrm{mol} / 1 \\
\mu \mathrm{mol} / 1 \\
\mu \mathrm{mol} / 1\end{array}$ & $\begin{array}{c}127,1 \\
2,977 \\
87,70 \\
47,37 \\
24,86 \\
0,449 \\
2,300 \\
1,841 \\
1,856 \\
37,58 \\
2,820 \\
125,7\end{array}$ & $\begin{array}{c}131,8 \\
3,171 \\
90,06 \\
49,74 \\
26,47 \\
0,472 \\
2,699 \\
2,033 \\
1,962 \\
42,54 \\
3,196 \\
140,9\end{array}$ & $\begin{array}{c}134,6 \\
3,408 \\
93,11 \\
53,42 \\
28,80 \\
0,539 \\
3,061 \\
2,607 \\
2,066 \\
48,41 \\
3,948 \\
168,2\end{array}$ & $\begin{array}{c}142,8 \\
4,351 \\
103,5 \\
70,01 \\
42,67 \\
1,123 \\
6,020 \\
5,490 \\
2,431 \\
85,08 \\
8,647 \\
313,5\end{array}$ & $\begin{array}{c}149,0 \\
5,637 \\
110,1 \\
81,73 \\
50,49 \\
1,704 \\
9,937 \\
19,47 \\
2,726 \\
240,6 \\
32,70 \\
532,1\end{array}$ & $\begin{array}{c}151,1 \\
6,177 \\
112,1 \\
84,16 \\
51,85 \\
1,914 \\
11,04 \\
27,43 \\
2,850 \\
701,0 \\
60,05 \\
590,2\end{array}$ & $\begin{array}{c}152,5 \\
7,370 \\
113,3 \\
86,97 \\
52,63 \\
2,144 \\
11,84 \\
31,24 \\
2,989 \\
932,4 \\
87,75 \\
664,4\end{array}$ \\
\hline
\end{tabular}


Tab. 5. Prozentuale Häufigkeit von Profilen mit Werten außerhalb der $95 \%$-Referenzbereiche im Referenzkollektiv (1976: 313 Personen, 1977: 342 Personen)

\begin{tabular}{|c|c|c|c|}
\hline \multirow{2}{*}{$\begin{array}{l}\text { Anzahl der } \\
\text { Parameter } \\
\text { außerhalb der } \\
95 \%-\text { Referenz- } \\
\text { bereiche }\end{array}$} & \multicolumn{3}{|c|}{ Prozentualer Anteil von Profilen } \\
\hline & 1976 gefunden & 1977 gefunden & erwartet \\
\hline $\begin{array}{r}1 \\
2 \\
3 \\
4 \\
5 \\
\geqslant 6\end{array}$ & $\begin{array}{l}26,8 \\
7,99 \\
4,47 \\
0,319 \\
0 \\
0\end{array}$ & $\begin{array}{l}27,5 \\
6,14 \\
3,80 \\
1,17 \\
0,292 \\
0,292\end{array}$ & $\begin{array}{c}34,1 \\
9,88 \\
1,73 \\
0,205 \\
0,0173 \\
0,0317\end{array}$ \\
\hline Gesamt & 39.6 & 39,2 & 46,0 \\
\hline
\end{tabular}

unter Zugrundelegung von 95\%-Referenzbereichen wie folgt:

$$
P(x)=\left(\begin{array}{c}
12 \\
x
\end{array}\right)(1-0,95)^{x} 0,95^{12-x}
$$

Hierbei ist $\mathrm{x}$ gleich der Anzahl der gleichzeitig falsch positiven Resultate und $\mathrm{P}$ die Wahrscheinlichkeit für das Auftreten dieser Anzahl. Der Gesamtanteil ergibt sich natürlich durch Summation.

Um die beobachteten Unterschiede auf Signifikanz zu prüfen, haben wir aus den Gesamthäufigkeiten für die Referenzpersonen Vertrauensgrenzen bei einer Irrtumswahrscheinlichkeit von 5\% berechnet (13). Für die beiden Kollektive ergaben sich der Reihe nach:

$\mathrm{p}_{1}<\mathrm{p}<\mathrm{p}_{2}$ mit $\mathrm{p}_{1}=34,34 \%$ und $\mathrm{p}_{2}=45,11 \%$ bzw. $\mathrm{p}_{1}<\mathrm{p}<\mathrm{p}_{2}$ mit $\mathrm{p}_{1}=33,62 \%$ und $\mathrm{p}_{2}=43,91 \%$.

Der Wert der Binomialverteilung (46,0\%) liegt nicht in diesen Intervallen. Die Unterschiede sind also auf dem 5\%-Niveau bzw. bei einseitiger Fragestellung auf dem 2,5\%-Niveau signifikant. Bei den Patienten sind die Unterschiede noch größer.

Die prozentuale Häufigkeit ,falsch positiver Resultate“ im Patientenkollektiv ist noch geringer als die im Referenzkollek tiv (Tab. 6). Hierbei handelt es sich natürlich

Tab. 6. Prozentuale Häufigkeit von Profilen mit Werten außerhalb der 95\%-Bereiche im Patientenkollektiv (1976: 4763 Patienten, 1977: 3423 Patienten)

\begin{tabular}{lccc}
\hline $\begin{array}{l}\text { Anzahl der } \\
\text { Parameter } \\
\text { außerhalb der }\end{array}$ & \multicolumn{3}{c}{ Prozentualer Anteil von Profilen } \\
95\%-Bereiche & 1976 gefunden & 1977 gefunden & erwartet \\
\hline 1 & 20,6 & 20,5 & 34,1 \\
2 & 6,47 & 7,16 & 9,88 \\
3 & 3,02 & 3,77 & 1,73 \\
4 & 1,45 & 1,67 & 0,205 \\
5 & 0,819 & 0,730 & 0,0173 \\
$\geqslant 6$ & 1,15 & 0,643 & 0,0317 \\
\hline Gesamt & 33,5 & 34,5 & 46,0 \\
\hline
\end{tabular}

nicht um falsch positive Werte im eigentlichen Sinne, da die Grenzen anhand der Patientendaten definiert wurden. Die theoretisch zu erwartenden Häufigkeiten sollten aber dieselben sein, wenn die Annahme einer Binomialverteilung zuträfe. Der Vergleịch beider Jahrgänge hingegen zeigt auch hier eine gute Übereinstimmung der prozentualen Häufigkeiten. Für dieses überraschende Ergebnis kommen vorwiegend zwei Ursachen in Betracht:

1. Die biologischen Kôrrelationen der zu einem Profil zusammengestellten Parameter untereinander und

2. die aus den Tabellen 1 bis 4 ersịchtliche beträchtliche Abweichung der einzelnen Verteilungen von der Normalverteilung.

Um zu prüfen, ob auch die erste Ursache für das vorliegende Profil zutrifft, wurden die Matrizen der RangKorrelationen in den vier Kollektiven berechnet. In den Tabellen 7 und 8 sind jeweils die Korrelationen beider Jahrgänge spiegelbildlich zur Hauptdiagonalen gegenübergestellt. Es zeigt sich, daß von den 66 jeweils möglichen Korrelationen einige besonders stark ausgeprägt sind: $z$. B. weisen die Kombinationen Gesamt-Eiweiß/ Albumin, Albumin/Calcium, Gesamt-Eiweiß/Calcium, Kreatinin/Harnșäure, Natrium/Chlorid, Harnstoff-N/ Kreatinin und Harnstoff-N/Harnsäure in mindestens zweien der vier Kollektive Korrelationskoeffizienten über 0,3000 auf. Vergleicht man die Korrelationskoeffiżienten dieser sieben genannten Parameterpaare in den Patienten- und Referenzkollektiven, so fällt auf, daß mit Ausnahme der Kombination Kreatinin/Harnsäure die Korrelationen in den Patientenkollektiven stärker ausgeprägt sind. Weiterhin ist bemerkenswert, dảß sämtliche mathematisch stark korrelierenden Kenngrößen auch biologisch sinnvolle Zusammenhänge repräsentieren. Die Ergebnisse zeigen deutlich, daß die eine Unabhängigkeit der Kenngrößen voraussetzende Binomialverteilung zu Aussagen führen muß, die beträchtlich von den tatsächlich vorliegenden Verhältnissen abweichen.

Tabelle 9 zeigt einen Vergleich zwischen je einem der Referenz- und Patientenkollektive. Die Gegenüberstellung erlaubt folgende Aussagen:

1. Bedingt durch die größere Streuung der Patientenwerte liegt der prozentuale Anteil von Werten außerhalb der angegebenen Quantile bei allen Parametern deutlich über demjenigen im Referenzkollektiv.

2. Bedingt durch die unterschiedlich ausgeprägte Asymmetrie der Verteilungen der Patientenwerte, ist der prozentuale Anteil von Werten unterhalb und oberhalb dèr angegebenen Quantile nicht gleich, sondern weist zum Teil erhebliche Differenzen auf. Dieses Verhalten ist besonders deutlich beim Chlorid, Gesamt-Eiweiß und Albumin, wo Werte unterhalb der Referenzbereiche gegenüber Werten oberhalb der Referenzbereiche wesentlich häufiger sind sowie beim anorganischen Phosphor, Choleșterin und Harnstoff- 
Tabelle 7. Rang-Korrelationen zwischen den Kenngrößen des SMA 12/60-Profils in zwei Referenzkollektiven (Linke Seite: $1976, N=313 /$ Rechte Seite: $1977, N=342$ )

Natrium Kalium Chlorid Ges.-Eiw. Albumin Anorg.P Cholest. Harnst.-N Calcium Kreatinin Bilirubin Harnsäure

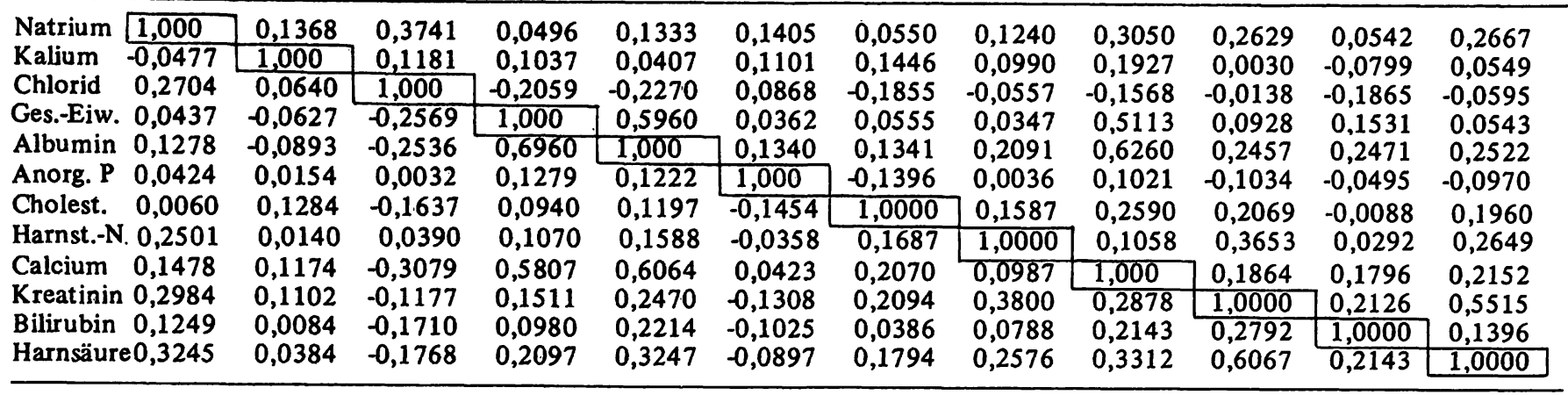

Tab. 8. Rang-Korrelationen zwischen den Kenngrößen des SMA 12/60-Profils in zwei Patientenkollektiven (Linke Seite: 1976, $N=750 /$ Rechte Seite: $1977, N=750$ )

\begin{tabular}{|c|c|c|c|c|c|c|c|c|c|c|c|c|}
\hline & Natrium & Kalium & Chlorid & Ges.-Eiw. & Albumin & Anorg. P & Cholest. & Harnst. -1 & $\checkmark$ Calcium & Kreatinin & n Bilirubin & Harnsäure \\
\hline $\begin{array}{l}\text { Natrium } \\
\text { Kalium } \\
\text { Chlorid } \\
\text { Ges.-Eiw. }\end{array}$ & \begin{tabular}{|l|}
1,0000 \\
0,0542 \\
0,3933 \\
0,1723
\end{tabular} & & & & & & & & & & & \\
\hline & $\begin{array}{r}0,3543 \\
-0,1381 \\
0,1776\end{array}$ & & & & & & & & & & & \\
\hline $\begin{array}{l}\text { Jarnst.-N } \\
\text { Calcium } \\
\text { Kreatinin } \\
\text { Bilirubin } \\
\text { Harnsäure }\end{array}$ & $\begin{array}{r}0,0180 \\
0,2601 \\
0,1069 \\
-0,0082 \\
e 0,1036\end{array}$ & $\begin{array}{r}0,1426 \\
0,2088 \\
0,2178 \\
-0,1620 \\
0,1457\end{array}$ & & $\begin{array}{r}-0,0560 \\
0,6623 \\
0,0618 \\
0,0930 \\
0,0865\end{array}$ & $\begin{array}{r}-0,1308 \\
0,6977 \\
0,0289 \\
0,1288 \\
0,0756\end{array}$ & $\begin{array}{r}-0,0427 \\
0,1069 \\
-0,1164 \\
-0,1158 \\
0,0352\end{array}$ & $\begin{array}{r}0,1542 \\
0,2442 \\
0,1654 \\
-0,0971 \\
0,1724\end{array}$ & $\begin{array}{r}1,0000 \\
-0,0296 \\
0,5324 \\
0,0773 \\
0,3427\end{array}$ & $\begin{array}{l}0,0601 \\
1,0000 \\
0,0599 \\
0,0273 \\
0,1477\end{array}$ & & $\begin{array}{l}0,0599 \\
0,0157 \\
0,1948 \\
1,0000 \\
0,1631\end{array}$ & $\begin{array}{l}0,3207 \\
0,1141 \\
0,4860 \\
0,1618 \\
1,0000 \\
\end{array}$ \\
\hline
\end{tabular}

Tab. 9. Häựigkeit von Werten im Patientenkollektiv $(N=3423)$ unterhalb bzw. oberhalb der Quantile des Referenzkollektivs $(\mathrm{N}=313)$

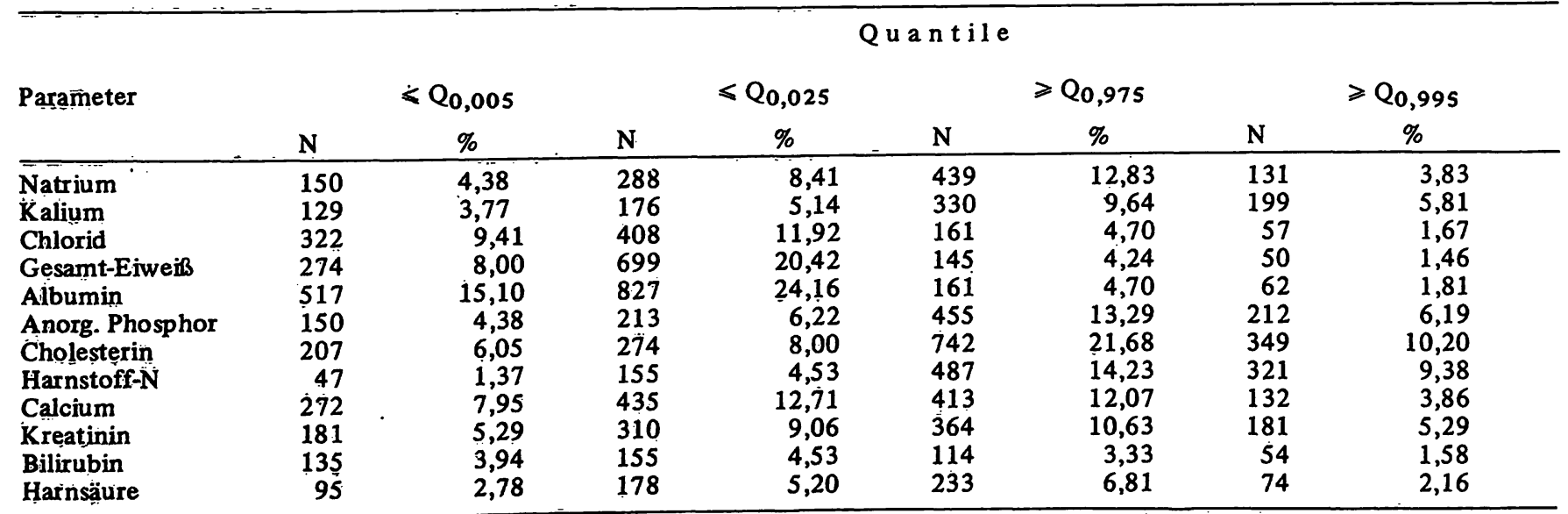


$\mathrm{N}$, die ein gegensätzliches Verhalten aufweisen.

Die Aussagen sind durchweg medizinisch sinnvoll: Hypoproteinämien sind wesentlich häufiger als Hyperproteinämien, Hypercholesterinämien z.B. im Rahmen von Fettstoffwechselstörungen häufiger als Hypocholesterinämien z. B. im Rahmen einer Hyperthyreose. Auch Harnstoff-N-Erhöhungen sind bei der Häufigkeit von Nierenerkrankungen in wesentlich größerer Zahl zu erwarten als Harnstoff-N-Erniedrigungen z. B. bei dem relativ selten vorkommenden Leberkoma und bei
Patienten, denen unmittelbar nach Dialyse Blut zur Untersuchung entnommen wurde.

Trotz der gefundenen Unterschiede in den Verteilungen erkennnt man, daß bei den einzelnen Kenngrößen die Uberlappungszonen bei Patienten und Referenzpersonen so groß sind, $d a ß$ im allgemeinen die Bestimmung einer einzelnen Kenngröße nicht ausreicht, um zwischen gesund und krank zu diskriminieren. Eine solche Diskriminierung könnte eventuell mit multivariaten Referenzbereichen $(14,15,16,17)$ erreicht werden.

\section{Literatur}

1. Eggstein, M. (1975), Med. Welt 26, 474-478

2. Kienle, G. (1978), Arztl. Lab. 24, 105-117

3. Kreutz, F. H. (1977), DIA 4, 93-103

4. Büttner, J. (1977), diese Z. 15, 1-12

5. Voigt, K. D., Harm, K., Domesle, A. \& Rehpenning, W. (1978), Technicon Symposium, Frankfurt a. M.

6. Voigt, K. D. \& Harm, K. (1979), Therapiewoche 29, (im Druck)

7. Reagent data handbook for the Technicon SMA $12 / 60$ system. Technical publication No. TH0-0160-10 (1971), Technicon Instruments Corporation, Tarrytown.

8. Technicon Information Bulletin No. TN3-0160-20 (1973), Technicon Instruments Corporation, Tarrytown

9. Klose, S., Hagen, A. \& Greif, H. (1976), in: Organisation des laboratoires et interprétation des résultats - Biologie prospective. Comptes rendus du troisième colloque international, Pont-à-Mousson 1975 (Siest, G., ed.), S. 505-507. L'Expansion Scientifique Française, Paris.
10. Harm, K. (1974), Med. Progr. Technol. 3, 45-55

11. Sachs, L. (1974), Angewand te Statistik, 4. Auflage, S. 75, Springer-Verlag, Berlin-Heidelberg-New York

12. Harm, K., Voigt, K. D., Rehpenning, W. \& Domesle, A. (1979), 4e Colloque International de Biọlogie Prospective, Pont-à-Mousson 1978 (im Druck)

13. Van der Waerden, B. L. (1957), Mathematische Statistik, Springer-Verlag, Berlin-G̈öttingen-Heidelberg

14. Bernhardt, W., Weisner, B. \& Rehpenning, W. (1978), diese Z. $16,435-439$

15. Buret, J., Monfort, F. \& Marthoz, J. (1976), in: Organisation des laboratoires et interprétation des résultats - Biologie prospective. Comptes rendus du troisième colloque international, Pont-à-Mousson 1975 (Siest, G. ed.), S. 751-754, L'Expansion Scientifique Française, Paris.

16. Grams, R. R., Johnson, E. A. \& Benson, E. S. (1972), Amer. J. Clin. Pathol. 58, 188-200

17. Winkel, P. (1972), Scand. J. Clin. Lab. Invest. 30, 339-344

Priv.-Doz. Dr. med. K. Harm Universitäts-Krankenhaus Eppend orf Zentrallabor der Med. Kliniken Martinistraße 52

D-2000 Hamburg 20 